\title{
Navigation System for Indoor Parking Based on Visible Light Communication
}

\author{
Qiang $\mathrm{Mai}^{1 *}$ and Chun-Liang $\mathrm{Hsu}^{2}$ \\ ${ }^{1}$ School of Electronics and Electrical Engineering, Dongguan Polytechnic, \\ No. 3, University Rd., Song-Shang Lake District, Dongguan, Guandong 523808, China \\ ${ }^{2}$ Department of Electrical Engineering, St. John's University, \\ No. 499, Sec. III, Tan-Kin Rd. Full, Tan-Shuei District, New-Taipei City 25135, Taiwan
}

(Received November 30, 2019; accepted April 20, 2020)

Keywords: visible light communication, indoor positioning, proximity method, fingerprint method

Parking difficulties are becoming increasingly prominent in cities with the rapid growth of car ownership and ineffective indoor position of global positioning system (GPS). We propose that visible light indoor positioning technology, with the use of LED lamps as communication equipment, can be applied to indoor parking lots to achieve high-precision navigation. In our system, the parking occupancy information is detected by reflective infrared sensors and modulated with light ID codes into LED lamps. PIN photodiode (PD) sensors receive light information and detect light intensity, then with the combined use of proximity and fingerprint methods to calculate the position coordinates, real-time positioning and navigation in indoor parking lots can be achieved. Our experiments have proved that when the LED light intensity is greater than 180 lux and the communication distance is less than $3 \mathrm{~m}$, the communication requirements can be met. Through continuous positioning trajectory testing, it was found that the combination of the proximity and fingerprint methods has higher positioning accuracy than the proximity method alone and can meet the needs of indoor parking positioning and navigation.

\section{Introduction}

With the rapid growth of car ownership in cities, the contradiction between the huge number of cars and limited parking spaces has become increasingly prominent, and the difficulty of parking has also been put forward as a significant public issue. Because of limited land in cities, underground and multilevel indoor parking has become the main solution to the problem of difficult parking. In recent years, indoor parking lots have sprung up, and car owners also tend to park indoors. However, without an efficient guidance system, owners can only park blindly, reducing the use efficiency of parking spaces. ${ }^{(1)}$ At present, the guidance of indoor parking lots is not very effective since it mainly displays spaces and directions through LED screens, so it is essential to design a system that can provide accurate indoor parking lot navigation services.

The rapid development of mobile Internet technology has led to the wide use of global positioning system (GPS) in many fields such as car navigation and map positioning, which

*Corresponding author: e-mail: maiq@dgpt.edu.cn

https://doi.org/10.18494/SAM.2020.2778 
has brought great convenience to people's travel and their daily life. However, when GPS is used inside buildings or underground, it is greatly affected by the reinforced concrete structure. Signal attenuation and interference will arise, causing ineffective positioning. Therefore, GPS navigation software cannot provide accurate indoor positioning and navigation. ${ }^{(2-4)}$ How to achieve low-cost, high-accuracy indoor positioning has become a research hotspot. At present, the commonly used indoor positioning technologies mainly use cameras, Wi-Fi, Bluetooth, ZigBee, etc. The positioning accuracy of camera image recognition positioning technology can reach more than $2.5 \mathrm{~m}$, but the cost is high, the algorithm is complex, the processing speed is relatively low, and it is easily affected by obstacles. Wi-Fi positioning accuracy can reach about $2 \mathrm{~m}$, but the fingerprint database construction requires a lot of work and time, and the signal is easily subjected to interference by the environment. Bluetooth and ZigBee need a large range of densely deployed new equipment for networking, and their positioning accuracy is about $3 \mathrm{~m}$. However, these technical solutions have the disadvantages of high difficulty in deployment, low accuracy, need for proprietary equipment, high cost, and susceptibility to electromagnetic wave interference, and cannot be directly applied to complex and changeable indoor environments. ${ }^{(5,6)}$ In view of these shortcomings, researchers have proposed a solution for indoor positioning, which can be realized by using LED visible light. LED lamps are used for scene lighting as communication equipment since they are simple to deploy and low in cost. Also, visible light signals are more powerful and stable than electromagnetic waves. Considering the above discussion, a navigation system for indoor parking based on visible light communication has been designed to achieve high-precision positioning without electromagnetic interference and simultaneously to realize lighting functions. ${ }^{(7-9)}$ Thus, it can be seen that the development and improvement of visible light indoor positioning technology have great implementation significance.

The principle of visible light communication technology is to encode and modulate information into LED lamps. The lights send information by blinking at high speeds. When the blinking frequency exceeds the recognition frequency of human eyes, the lights appear to be always on, so visible light communication technology can satisfy the needs of both normal lighting and information transmission. ${ }^{(10)}$ The technology is applied to an indoor positioning system, in which LED lamps are used to emit light signals, photodiode (PD) sensors are used to receive the light signals, a geometric model is selected according to different positioning algorithms, and the target position coordinates are calculated using the geometric relationship between the LED lamps and the target to be located.

In this paper, we describe how to build an indoor parking navigation system with the use of fixed LED lamps in indoor parking lots. The system comprehensively utilizes infrared sensor technology, PD sensors, LED modulation driving, and communication network technology to build a more accurate and efficient indoor parking navigation system. The main achievements of this system are as follows.

(1) A navigation method that takes into account both indoor positioning and real-time broadcasting of parking space information is proposed. The system uses a reflective infrared sensor to detect parking occupancy and modulate the space information and light ID codes into LED lamps. The receiver module updates and displays the vacant parking space 
on the map after receiving the light signal via the PIN PD, and accordingly, the positioning information and driving path are updated to realize vehicle positioning and navigation.

(2) An improved positioning algorithm based on the combination of the proximity and fingerprint methods is proposed. After light information is received through the PIN PD sensor and the light intensity is detected, the fingerprint method can be used to realize navigation when the light ID codes cannot be identified. Therefore, the proposed algorithm can improve the applicability and accuracy of the positioning system in an actual environment.

\section{System Working Principle and Structure}

\subsection{System working principle}

According to the visible light communication principle, the LED lamps of indoor parking lots can send unique light ID codes and parking space information through the light. When the vehicle, equipped with a receiver with a $\mathrm{PD}$, travels to the range illuminated by the LED light, it will receive the ID and parking information. The navigation software, based on the ID and light intensity, will use the positioning algorithm to match the location information and then locate it on the parking map. At the same time, the vacant parking space on the map will be updated in real time for car owners to choose from. When the owner selects a vacant parking space, the software will plan the optimal path on the basis of the navigation algorithm. When the vehicle is driven under different LED lights along the path, the positioning information will be updated accordingly to complete the navigation function. If the selected vacant parking space becomes occupied by another car as it is approached, the system will update its navigation path and adjust to an adjacent parking space.

\subsection{System structure}

The indoor parking lot navigation system is shown in Fig. 1. The system is composed of a parking space management system, LED lamps with a unique light-coded ID, and a light signal receiver module. The management system processes the detection signals from the reflective infrared sensor installed on the parking space through the server and sends the parking space information to the LED lamps through the ZigBee wireless communication module. After

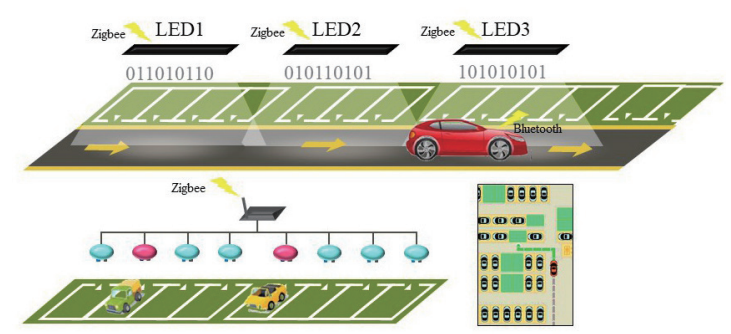

Fig. 1. (Color online) System framework. 
receiving the space information, the LED lamps encode and send the parking space information and the light ID codes as light signals. When the light signal receiver module receives the signals through the PIN PD, the signals will be amplified, filtered, and compared, and then decoded by the decoding circuit. Then, the parking space information and its ID are sent to the user terminal by the Bluetooth module; the terminal keeps updating the vacant parking space information and driving path on the map to realize indoor positioning and navigation.

\section{Positioning Model and Algorithm for Indoor Parking Lot}

\subsection{Positioning model and LED light intensity distribution}

The layout of the indoor parking lot positioning system model designed in this research is shown in Fig. 2. The dimensions of the parking lot are $4 \mathrm{~m} \times 3 \mathrm{~m} \times 2 \mathrm{~m}$. The system consists of an LED light signal transmitting end and a PD receiving end. Two $7 \mathrm{~W}$ LED lamps are installed at a height of $2 \mathrm{~m}$ and an interval of $3 \mathrm{~m}$.

To determine the light intensity distribution of the model, the collection points are determined as $X Y$ coordinates in units of $25 \mathrm{~cm}$. The light intensity of the model is measured by a light meter. As shown in Fig. 3, the maximum light intensity is directly below each LED; the light from the two LEDs overlaps and the light intensity of each collection point is different. This also lays the foundation for using the fingerprint method for position matching.

\subsection{Proximity and fingerprint method for indoor positioning}

In the visible light indoor positioning system, the actual position of the target is mainly calculated through various positioning algorithms. The positioning principle is that the light signal with position information is encoded and transmitted by the fixed LED lamps; the

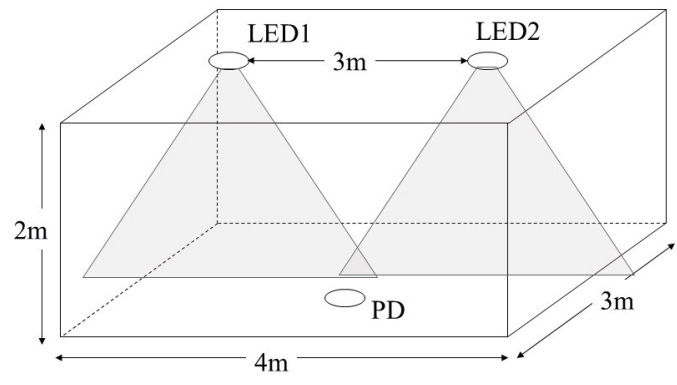

Fig. 2. Layout of indoor parking lot positioning system model.

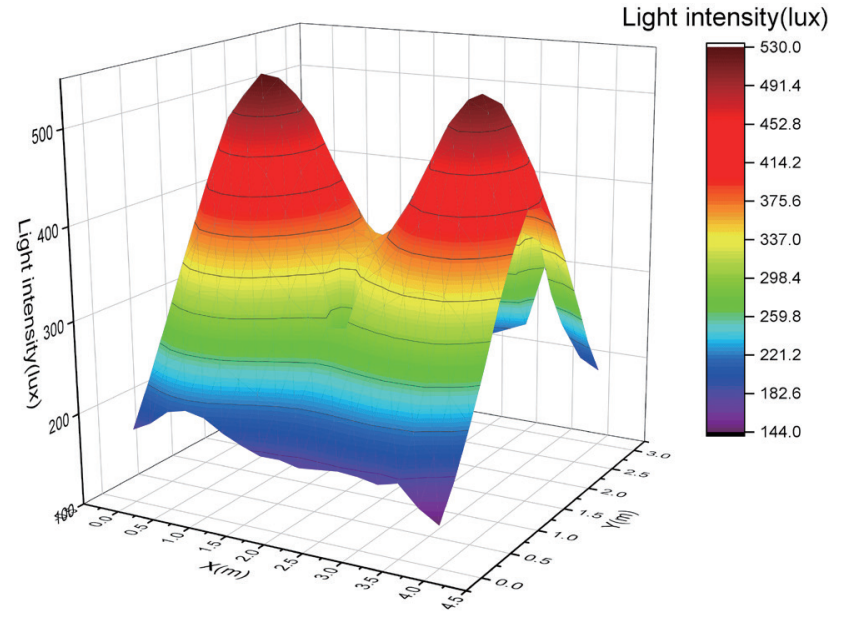

Fig. 3. (Color online) Light intensity distribution of double LED. 
receiving end gets the light signal through the PD sensor and performs amplification, decoding, and demodulation to restore the position signal, then it calculates and analyzes the position coordinates with the application of a positioning algorithm.

The common algorithms for visible light indoor positioning mainly include the proximity method, fingerprint method, time of arrival (TOA) method, time difference of arrival (TDOA) method, received signal strength (RSS) method, and angle of arrival (AOA) method. ${ }^{(11)}$ Under actual conditions, the LED lamps for an indoor parking lot are mainly distributed on the driveway, and there is a certain distance between the driveways, so the LED lights do not overlap. As shown in Fig. 4, the distribution of LED lights in indoor lots can be considered linear. Each lamp is installed at a certain distance. In order to ensure the continuity of lighting, two adjacent lights have to maintain a certain overlap. Because of the LED lamp distribution and the vehicle's one-way forward movement, the combined use of the proximity and fingerprint methods is chosen to complete the calculation of position coordinates.

The proximity method, also called the ID method, is the simplest positioning algorithm. In this method, each LED lamp has a unique ID, and its position is fixed and known. By matching the ID with the corresponding LED lamp position, position information without an ID can be obtained. When the receiving end approaches a certain LED lamp, it will receive the corresponding ID, allocate it to the required position through the ID, and estimate the position as the location of the receiving end. ${ }^{(12-15)}$ In the proximity method, spaces in the illuminated area of the same LED are considered as the same position, and the accuracy will be limited by the light density, which can be used in scenarios with lower accuracy requirements. Thus, in indoor parking lots, considering the vehicle's speed and the distribution density of LED lights, the proximity method can be used to meet the user's needs for indoor parking positioning.

In the proximity method, the transmitting end and the receiving end perform straight-line transmission without obstruction, which is usually called the light of sight (LOS) channel. Its typical transmission model is Lambert's model as shown in Fig. $5 .^{(16,17)}$ When only the LOS

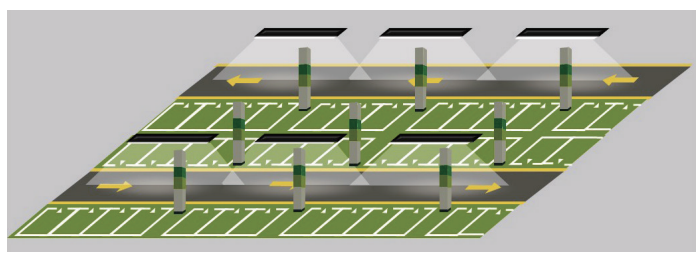

Fig. 4. (Color online) Model of indoor parking LED light distribution.

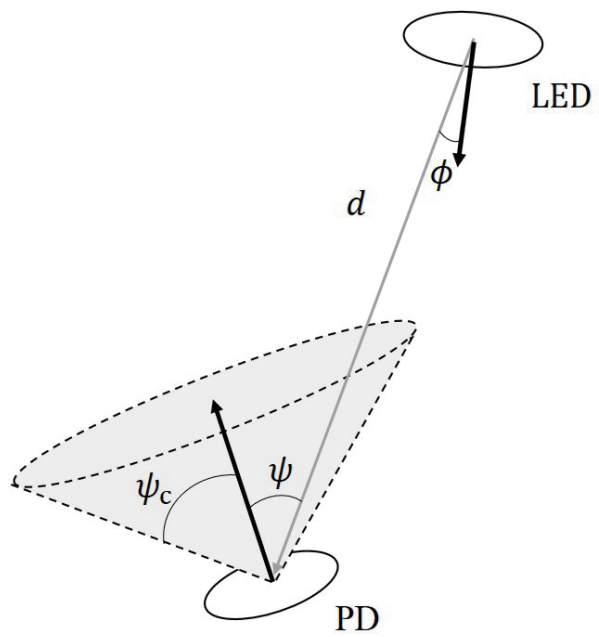

Fig. 5. Visible light transmission between LED and PD. 
channel is taken into consideration, the DC gain $H(0)$ from the transmitting end to the receiving end can be expressed as

$$
H(0)=\left\{\begin{array}{lc}
\frac{m+1}{2 \pi}\left(\frac{A}{d^{2}}\right)\left(\cos ^{m}(\phi) T(\psi) \cos (\psi) g(\psi)\right), & 0 \leq \psi \leq \psi_{c} \\
0 . & \psi \geq \psi_{c}
\end{array}\right.
$$

Here, $m$ is Lambert's order of the LED, which characterizes its light-emitting directivity and can be calculated from the half-power angle of the LED: $m=-\log 2 / \log (\cos (1 / 2))$; A represents the actual effective receiving area from the $\mathrm{PD} ; d$ refers to the distance between the LED emitting end and the PD; $\phi$ is the emission angle, the angle between the light emission direction and the LED normal direction; $\psi$ is the incident angle, the angle between the light incidence direction and the PD normal direction; $\psi_{c}$ represents the field of view (FOV) of the PD, which is the maximum incident angle that the PD can accept; $T(\psi)$ is the gain of the optical filter at the receiving end; and $g(\psi)$ is the gain of the optical condenser, which can be calculated as Eq. (2).

$$
g(\psi)= \begin{cases}\frac{n^{2}}{\sin ^{2} \psi_{c}}, & 0 \leq \psi \leq \psi_{c} \\ 0 . & \psi \geq \psi_{c}\end{cases}
$$

The DC gain value of the LOS channel shifts increasingly farther away from the LED center at the receiving end, so the receiver can accurately receive the LED light signal at a point near vertically below the LED light. When the receiver is away from the vertical center, signals in the overlapping area of two adjacent LED lights may interfere with each other and communication may not be performed normally. To better solve the problem that the LED light overlap area cannot be located, the fingerprint method is used to locate the area to improve the positioning accuracy.

The fingerprint method is also called the scene analysis method. This algorithm uses one or more signal features as fingerprints and uses the correlation between the fingerprint and the receiver's position to locate parking spaces. In visible light indoor positioning, the intensity of the received light signal is the visible light signal feature that is most commonly used as a fingerprint. The positioning process of the fingerprint method mainly consists of two stages: ${ }^{(18)}$ (1) Fingerprints are collected to establish a database of location fingerprints. Some positions are usually selected as reference points in the positioning area and the light intensity is collected as fingerprints at each reference point. The position fingerprint record is formed with the position coordinates of the reference point and then stored in the position fingerprint database. The flow chart of the offline training phase is shown in Fig. 6.

(2) The signal features measured by the receiver are matched with the fingerprints in the position fingerprint database, and then the target position can be estimated by a matching algorithm. Figure 7 shows the flow chart of the online positioning phase.

The major advantage of the fingerprint method is that the positioning is achieved by matching actual measured values with premeasured fingerprints without being affected by 


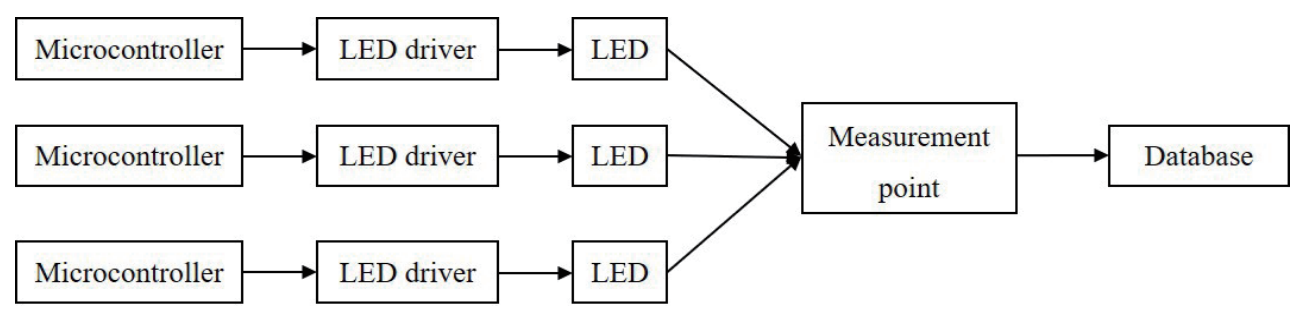

Fig. 6. Off-line flow chart of positioning phase.

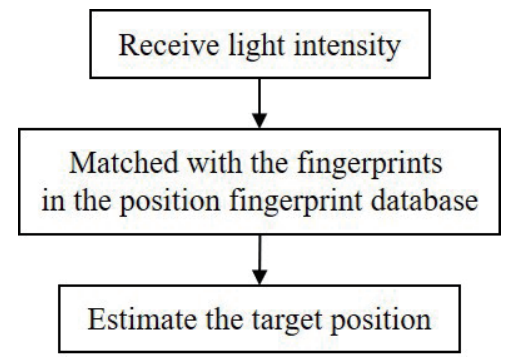

Fig. 7. On-line flow chart of positioning phase.

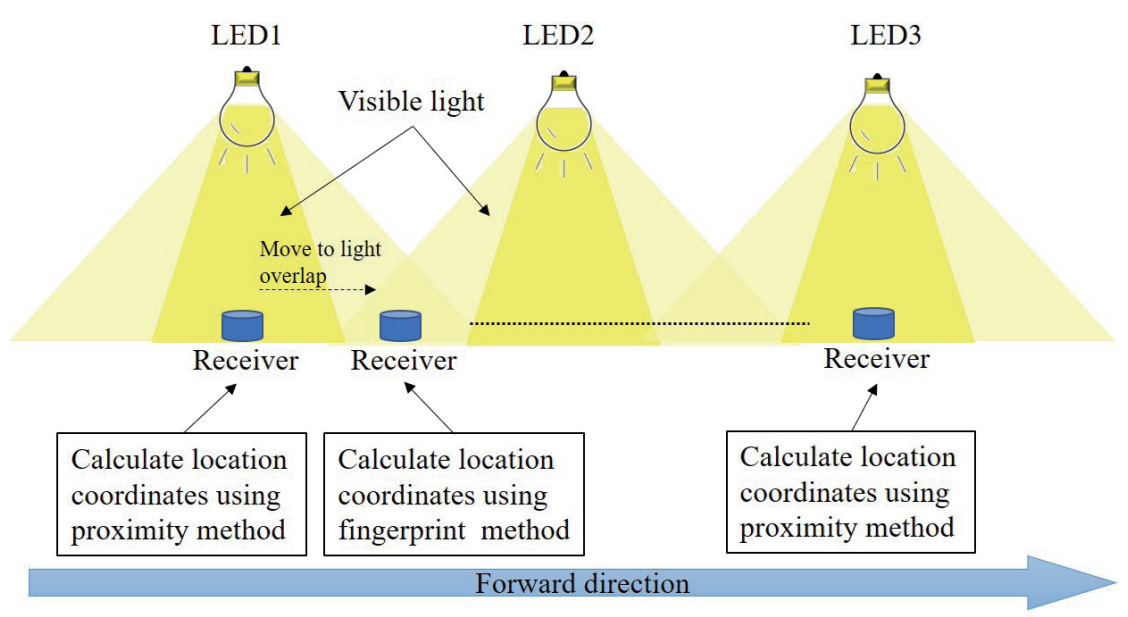

Fig. 8. (Color online) Scene of indoor parking lot positioning.

the site or requiring a large amount of calculation. However, the fingerprint method needs to establish a position fingerprint database in advance. If the scene area is large, establishing the database will be very time-consuming and labor-intensive.

The scene of the indoor parking lot is shown in Fig. 8. The proximity method is adopted within the vertical center of the LED lights, and the fingerprint method is adopted in the overlapping area of the two LED lights. When the reference-point fingerprints in the overlapping area have been collected in advance, the proximity and fingerprint methods can be combined to achieve positioning. This can solve the problem that the proximity method cannot receive the positioning ID in the area where the lights overlap, and it can reduce the number of fingerprint collections and improve the matching speed. 


\section{System Hardware and Software Techniques}

\subsection{Hardware design of parking space management system}

The block diagram of the parking space management system is shown in Fig. 9. The system consists of a parking space detection module, a signal processing module, and a ZigBee wireless communication module.

A reflective infrared sensor, which is composed of an infrared emitting diode and a infrared acceptor, is used to detect whether a parking space is occupied or not. The infrared emitting diode emits infrared light. If the parking space is occupied, the infrared light will be reflected and received by the infrared acceptor and output a low level after comparison processing; otherwise, the infrared light will not be received by the infrared acceptor if the parking space is vacant, and it will output a high level after comparison processing.

STC15 microcontrollers detect the output signal of the reflective infrared sensor through the I/O port, use eight parking spaces as one data, store the parking space information in an array form, and send the parking space array information through the serial port to the ZigBee module for broadcasting.

The ZigBee CC2530 module adopts a broadcast mode for wireless communication. Since the indoor parking lot is positioned and illuminated by LED lamps, the parking space management system and LED lamps use the form of ZigBee networking. The parking space information is broadcast through the ZigBee module which, after receiving the broadcast signal, will update the vacant parking space information.

\subsection{Hardware design of LED lamps}

After the LED lamps receive the parking space information from the parking management system through the ZigBee module, the STC15 microcontroller encodes the parking space information with pre-assigned unique light ID codes and then uses the LED driving circuit to switch on and off (OOK) the LED lamps to transmit the visible light communication positioning signal. The structural block diagram of the LED lamps is shown in Fig. 10.

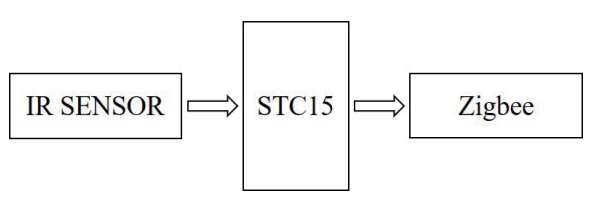

Fig. 9. Block diagram of parking space management system.

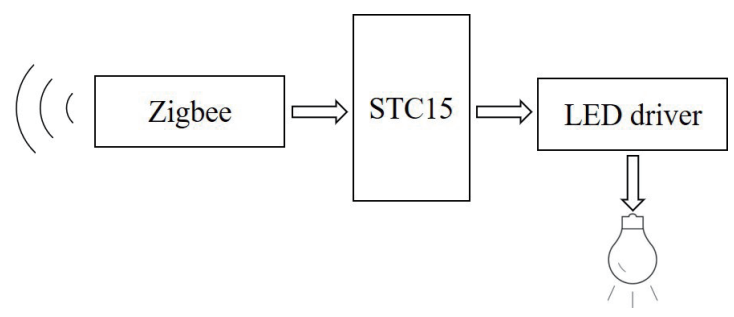

Fig. 10. Block diagram of LED lamps. 


\subsection{Hardware design of the light signal receiver}

The light signal receiver uses a PIN PD sensor to receive the light signal sent by the LED lamps. After $I-V$ conversion and amplification, the signal is processed by the LM393 comparison circuit and then input to the STC15 microcontroller for decoding. The parking space and location information are transmitted by the Bluetooth module to the user terminal. The block diagram of the light signal receiver structure is shown in Fig. 11.

\subsection{Coding and modulation method of visible light communication}

LED lamps are mainly used in indoor parking lot navigation and positioning systems to send light-coded IDs and parking space information. The data transmission rate is not required to be high. Therefore, an OOK modulation method is adopted, and the LED is turned on and off to realize data transmission.

In order to better meet the functions of lighting and data transmission, it is necessary for LED lamps to avoid flickering, which causes discomfort to human eyes. According to the IEEE standard, when the frequency is greater than $200 \mathrm{~Hz}$, human eyes cannot detect any flicker, but in OOK modulation, if the BCD coding method is used, 0 and 1 of different lengths will appear in the data, resulting in fluctuations in the light intensity and producing flicker. ${ }^{(19)}$ The differential Manchester encoding method is adopted to solve the problem of flicker caused by encoding. As shown in Fig. 12, there is a transition in the middle of each bit. The transition from high to low indicates "1", and the transition from low to high indicates " 0 ". In each digital signal coding, there are 0 and 1 of the same duration, thereby avoiding flicker and discomfort to human eyes.

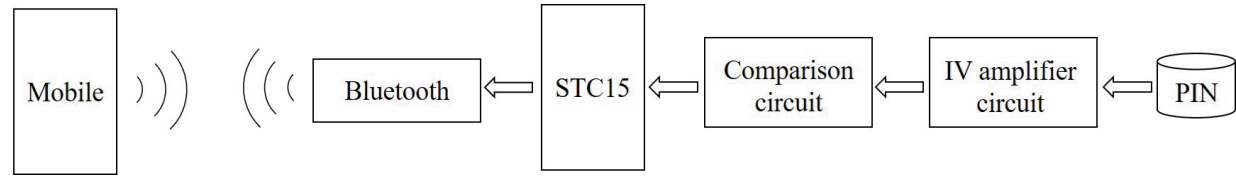

Fig. 11. Light signal receiver.

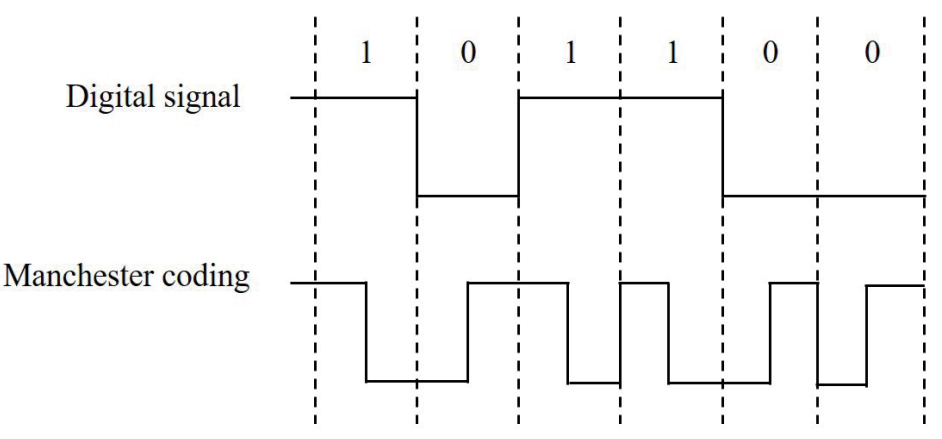

Fig. 12. Transmitting and receiving waveforms. 


\section{Experiment and Discussion}

\subsection{Visible light communication distance test}

In an indoor parking lot, under the premise of both lighting and communication, it is necessary to keep the light intensity moderate so that human eyes will not feel discomfort. As shown in Fig. 13, 1000 data signals are randomly sent, and the relationship between the bit error rate and the light intensity is calculated from the receiving end's conditions. The experimental results show that when the light intensity is less than 180 lux, the bit error rate is significantly increased and cannot meet the needs of communication.

Figure 14 shows the signals transmitted and received by the oscilloscope when the distance between the LED transmitting and receiving ends is $2.8 \mathrm{~m}$. In the experiment, $4 \mathrm{~W}$ LED lamps are used, and the maximum communication distance is $3 \mathrm{~m}$, which can meet the positioning requirements in most parking lots.

\subsection{Continuous positioning trajectory test}

To observe the effect and simulate the real-time positioning in practical applications, it is necessary to test the positioning accuracy of continuous walking for some time.

The corridor shown in Fig. 15 is selected as the test section. LED lamps for positioning are installed at intervals of $5 \mathrm{~m}$. The handheld light signal receiving end walks at a uniform speed. The position coordinates of the system and the actual position coordinates are recorded every $3 \mathrm{~m}$ for comparison and analysis.

Figure 16 shows a comparative analysis of the position calculation test using the proximity method, with which the test position trajectory and the actual position can be found. Within the vertical lighting range of the LED lamp, its corresponding position coordinates can be located. However, after leaving the area, they cannot be located.

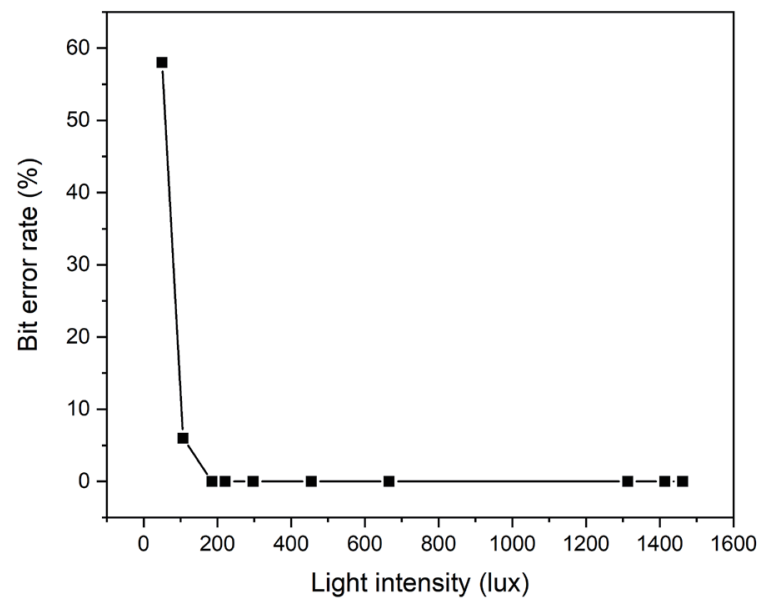

Fig. 13. Relationship between bit error rate and light intensity.

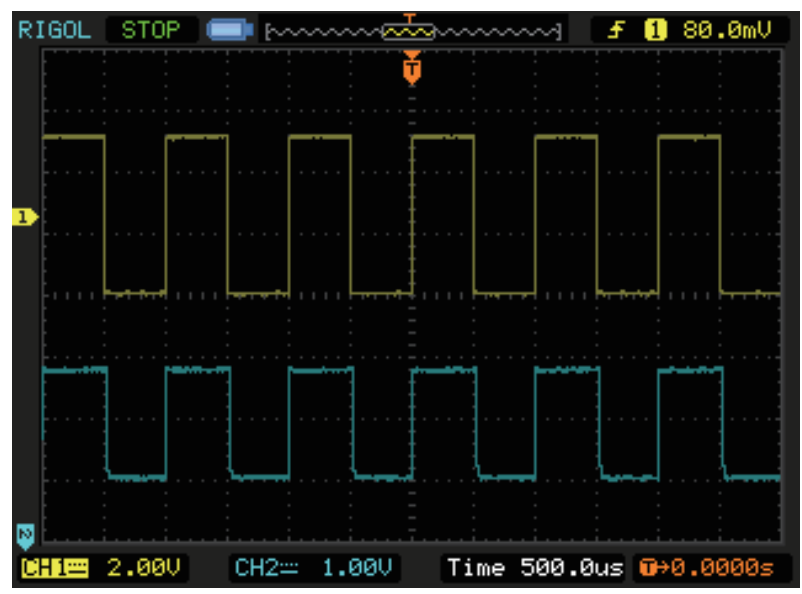

Fig. 14. (Color online) Transmitting and receiving waveforms. 

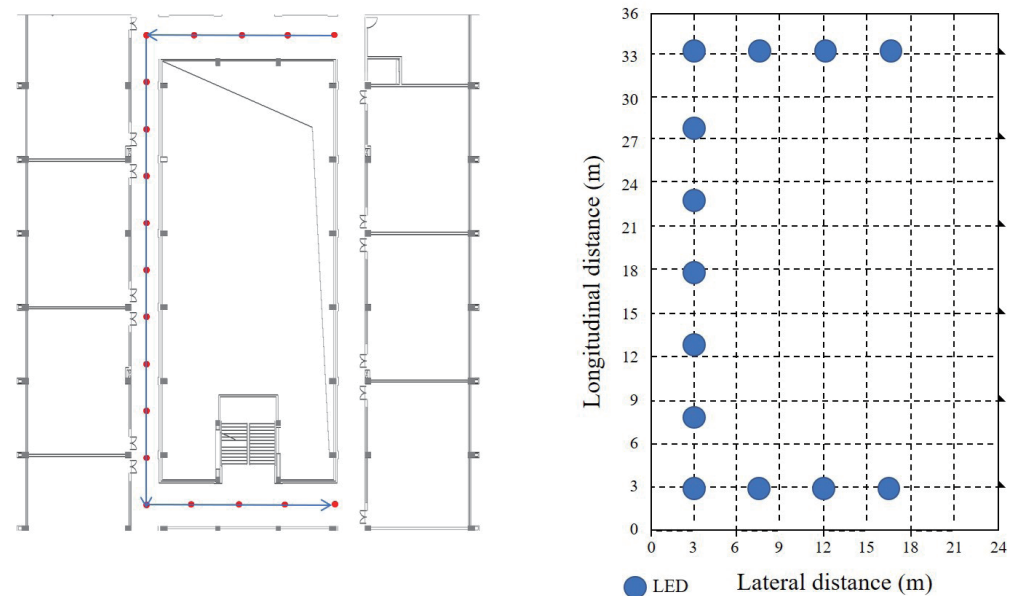

Fig. 15. (Color online) Positioning trajectory test route.

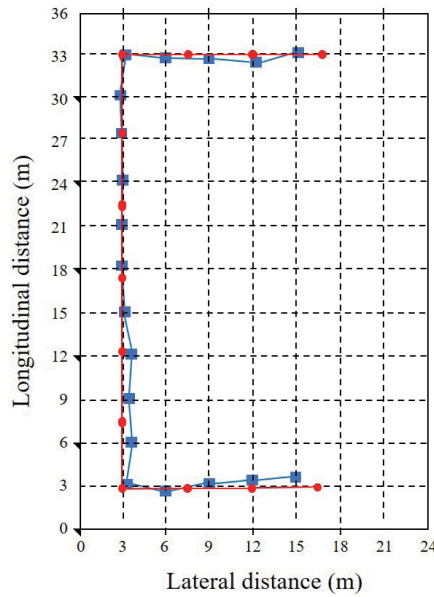

Fig. 16. (Color online) Test results of position calculation using the proximity method.

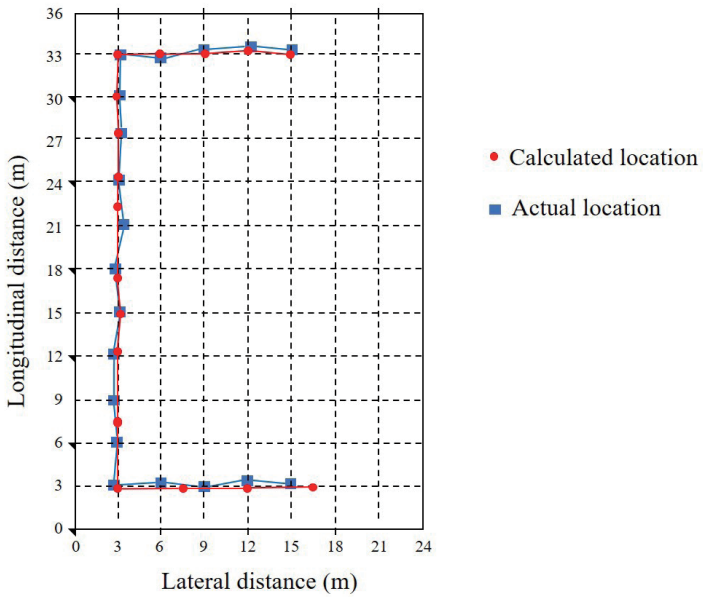

Fig. 17. (Color online) Test results of position calculation using proximity and fingerprint methods.

Figure 17 shows a comparative analysis of the position calculation test with the proximity method and the fingerprint method. The deviation between the test position trajectory and the actual position is within the range of $0.2-2.5 \mathrm{~m}$, and there is no situation of no positioning or large deviation during the test, which illustrates that the stability of the system is improved compared with that when using only the proximity method.

\section{Conclusion}

Visible light indoor positioning technology is applied to positioning and navigation for indoor parking lots, where sensor and communication technology is used to send parking space information and light ID codes through LED lights. It is verified that when the LED light is greater than 180 lux, it can accurately receive the light information and realize the real-time 
path navigation function; the combined use of the proximity and fingerprint methods is adopted to calculate the position coordinates to reduce the fingerprint database construction time, and the required positioning accuracy is greater than $2.5 \mathrm{~m}$. In this way, it effectively solves the problems that GPS navigation cannot be used indoors and it is difficult for owners to find parking spaces and to get their cars back.

\section{Acknowledgments}

This research was funded by Project of Dongguan Polytechnic (grant number 2018a10), Innovation and Entrepreneurship Training Program for College Students of Dongguan Polytechnic (grant number DC201901), and Dongguan Social Science and Technology Development Project.

\section{References}

1 S. Xu, Z. Wang, H. Zhang, and S. Sam: Sens. Mater. 31 (2019) 2043. https://doi.org/10.18494/SAM.2019.2253

2 J. M. Nocquet: Tectonophysics 579 (2012) 220. https://doi.org/10.1016/j.tecto.2012.03.037

3 Y. Yu, X. Xue, N. Zou, and J. Wang: Opt. Commun. Technol. 4 (2018) 51 (in Chinese). https://doi.org/10.13921/ j.cnki.issn1002-5561.2018.04.014

4 Q. Xu, X. Li, and C. Y. Chan: Sens. 17 (2017) 1431. https://doi.org/10.3390/s17061431

5 C. Chen and W. Tan: Comput. Eng. Appl. 5 (2015) 82 (in Chinese). https://doi.org/10.3778/ j.issn.1002-8331.1401-0025

6 J. Li, J. Mo, J. Fang, P. Lin, Z. Chen, Q. Lyu, F. Liang, H. Zheng, Y. Zhong, J. Fang, and Z. Chen: J. Appl. Opt. 40 (2019) 746 (in Chinese). https://doi.org/10.5768/JAO201940.0501005

7 Z. Zhou, M. Kavehrad, and P. Deng: Opt. Eng. 51 (2012). https://doi.org/10.1117/1.oe.51.8.085009

8 J. Armstrong, Y. A. Sekercioglu, and A. Neild: IEEE Commun. 51 (2013) 68. https://doi.org/10.1109/ MCOM.2013.6685759

9 J. K. Park, T.-G. Woo, M. Kim, and J. T. Kim: IEEE Photonics J. 9 (2017) 1. https://doi.org/10.1109/ JPHOT.2017.2667038

10 C.-W. Hsu, J.-T. Wu, H.-Y. Wang, C.-W. Chow, C.-H. Lee, M.-T. Chu, and C.-H. Yeh: IEEE Photonics J. 8 (2016) 1. https://doi.org/10.1109/JPHOT.2016.2590945

11 Y.-W. Ma, J.-L. Chen, and Z.-Z. Chen: Trans. Emerging Telecommun. Technol. 30 (2019) 3452. https://doi. org/10.1002/ett.3452

12 I. Lee, M. Sim, and F. Kung: Proc. 2008 4th IEEE Int. Conf. Circuits and Systems for Communications (IEEE, 2008) 698. https://doi.org/10.1109/ICCSC.2008.153

13 G. Campo-Jimenez, J. Perandones, and F.J. Lopez-Hernandez: Proc. 2013 Int. Conf. Localization and GNSS (IEEE, 2013) 1. https://doi.org/10.1109/ICL-GNSS.2013.6577276

14 S. Ayub, B. Honary, S. Kariyawasam, and M. Honary: Proc. 2013 5th IET Int. Conf. Wireless, Mobile and Multimedia Networks (ICWMMN 2013). https://doi.org/10.1049/cp.2013.2419

15 M. Nakajima and S. Haruyama: Proc. 2012 1st IEEE Int. Conf. Communications (IEEE, 2012) 524. https://doi. org/10.1109/ICCChina.2012.6356940

16 H. Wei and Y. Hao: Proc. 2017 IEEE 2nd Advanced Information Technology, Electronic and Automation Control Conf. (IEEE, 2017) 2412. https://doi.org/10.1109/IAEAC.2017.8054455

17 C. Chen, W.-D. Zhong, and D. Wu: J. Opt. Commun. Networking 9 (2017) 36. https://doi.org/10.1364/ JOCN.9.000036

18 S. Yang, E. Jeong, and S. Han: Electron. Lett. 50 (2014) 49. https://doi.org/10.1049/el.2013.2944

19 S. Rajagopal, R. D. Roberts, and S.-K. Lim: IEEE Commun. Mag. 50 (2012) 72. https://doi.org/10.1109/ MCOM.2012.6163585 


\section{About the Authors}

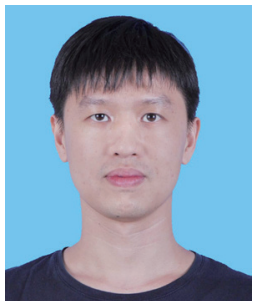

Qiang Mai received his B.S. degree from Henan University of Science and Technology, China, in 2006 and his M.S. degree from Sun Yat-Sen University, China, in 2009. Since 2010, he has been a lecturer at Dongguan Polytechnic. His research interests are in optical technology and microcontrollers.

(maiq@dgpt.edu.cn)

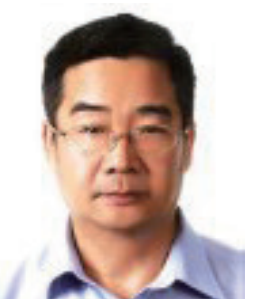

Chun-Liang Hsu received his B.S. degree from National Taiwan Normal University, Taiwan, in 1977 and his M.S. and Ph.D. degrees from the Industry Education Institute, Taiwan, in 1980 and 2000, respectively. From 2000 to 2009, he was an assistant professor at St. John's University, Taiwan. Since 2010, he has been a professor at St. John's University. His research interests are in microsystem technology, embedded systems, and wireless communication and sensors. (liang@mail.sju.edu.tw; xucl@dgpt.edu.cn) 